\title{
Interaction of polymorphonuclear leucocytes with patellar cartilage of immobilised arthritic joints: a scanning electron microscopic study
}

\author{
P L E M VAN LENT, ${ }^{1}$ F H A WILMS, ${ }^{2}$ AND W B VAN DEN BERG
}

From the ${ }^{I}$ Department of Rheumatology, University Hospital, St Radboud, Geert Grooteplein Zuid 8, 6525. GA Nijmegen, The Netherlands; and the ${ }^{2}$ Department of Botany, University of Nijmegen, Toernooiveld, $6525 \overrightarrow{\vec{\omega}}$ ED Nijmegen, the Netherlands

SUMMARY Short term immobilisation (seven days) of an antigen induced arthritic knee joint of the mouse led to attachment of polymorphonuclear leucocytes to the cartilage surface and severe $e_{\infty}^{\circ}$ cartilage damage. The events at the patellar cartilage surface were studied by scanning electron microscopy and compared with the changes found in non-immobilised arthritic joints. At day 70 the immobilised arthritic joint showed an articular cartilage which was covered by a mat of fibrinous material and cell remnants. Moreover, inflammatory cells, clearly attached to the cartilage, showed large protrusions of their membranes. The cartilage surface, furthermore $\frac{?}{0}$ showed numerous fissures of varying size and depth. These cartilage changes were much more足 pronounced in immobilised arthritic joints than in non-immobilised arthritic joints, and clear cellt adhesion was exclusively found in the former. These findings show that immobilisation $\$$ inflamed mouse knee joints leads to increased damage and thus although clinical immobilisation is used to relieve pain, this may also risk damage to the articular cartilage.

Joints of patients with rheumatoid arthritis are sometimes immobilised to relieve pain during an exacerbation. Published reports do not indicate whether short term immobilisation ameliorates cartilage damage or causes deterioration. In rheumatoid arthritis, cartilage damage is often observed. The part played by polymorphonuclear cells (PMNs) in cartilage destruction is controversial, some investigators considering its role to be minor ${ }^{12}$ and others major. ${ }^{34}$ Polymorphonuclear cells, lying in the cavity and synovial structures of arthritic joints, release degradative enzymes 5 and toxic oxygen metabolites, ${ }^{67}$ which may cause damage to the cartilage matrix and the chondrocytes. The trigger which activates the PMNs to release these degradative products is not known. It is suggested by many investigators that immune complexes, which are present in large amounts in rheumatoid arthritis synovial fluids and cartilaginous tissues, are responsible for this PMN activation. ${ }^{4}$ Immune com-

Accepted for publication 10 March 1989.

Correspondence to Dr P L E M van Lent. Department of Rheumatology, University Hospital, St Radboud, Geert Grooteplein Zuid 8, 6525 GA Nijmegen. The Netherlands. plexes deposited in the superficial layers may play part in the so called frustrated phagocytosis by PMNs which attach to the cartilage surface. The attachment of PMNs to cartilaginous tissues is onlys observed, however, in vitro. ${ }^{89}$ In vivo such attach ment is seen only rarely.

Cartilage destruction seen in rheumatoid arthritis resembles cartilage destruction observed in allergico arthritis models in mice ${ }^{10} 11$ and rabbits. ${ }^{12}$ For this reason we used the antigen induced arthritis mode $B$ to study the effect of short term immobilisation on cartilage destruction. A severe chronic arthritis was induced by intra-articular injection of cationic bovine serum albumin in previously immunised? mice. ${ }^{10}$ Firstly, we investigated over a period of time the surface topography of the articular cartilage with the scanning electron microscope. For this purposen the anatomically intact patella, which is easily dissected from the joint, was isolated $1,3,7$, and 140 days after arthritis induction. Secondly, in aे separate group (all groups consisted of at least five animals) the arthritic joint was immobilised fot? seven days and the cartilage surface of the patellato was compared with those derived from none immobilised arthritic joints. 


\section{Materials and methods}

A N I M A L S

Male C57 bl/10 mice aged 6-8 weeks at the start of the experiment were used. They were fed a standard diet and tap water freely.

\section{CHEM I C A L S}

Bovine serum albumin (fraction V) and 1-ethyl-3-(3dimethylaminopropyl)carbodiimide were obtained from Sigma Chemical Company, St Louis, Mo, USA. $N, N$-dimethy-1,3-propanediamine was obtained from BDH Chemicals Ltd, Poole, England.

BOVINE SERUM ALBUMIN MODIFICATION

Bovine serum albumin was modified according to the method of Danon et al, ${ }^{13}$ using 1-ethyl-3-(3dimethylaminopropyl)carbodiimide as an activator and $N, N$-dimethy-1,3-propanediamine as a nucleophile, as described previously. ${ }^{10}$ In this way free carboxyl groups of the protein were coupled to amino groups of $N, N$-dimethy-1,3-propanediamine. The isoelectric point was thus raised.
I M M OBILISATION PROCEDURE

Immediately after injection of cationic bovine serum albumin into the knee joint cavity the hind leg was immobilised in extension. The lower limb of the mouse was turned with plaster. Subsequently, a 3 $\mathrm{cm}$ long upper part, cut from a $5 \mathrm{ml}$ plastic tip, was pushed over the hind limb. The foot was fixed to the plastic immobilisation with a small piece of tape. At the end of the experiment the tape was easily removed from leg and plastic tip with $70 \%$ acetone. $^{14}$

\section{IMMUNISATION AND INDUCTION OF A R T H R I T IS}

Mice were immunised with $100 \mu \mathrm{g}$ amidated bovine serum albumin in $0.1 \mathrm{ml}$ Freund's complete adjuvant emulsion as previously described. ${ }^{10} \mathrm{On}$ day 21 after immunisation a unilateral arthritis was induced by intra-articular injection of $60 \mu \mathrm{g}$ of cationic bovine serum albumin in $6 \mu$ linto the right knee joint. No infection of the injected knee joints was ever observed. On days $1,3,7$, and 14 after arthritis induction at least five mice were anaesthe-
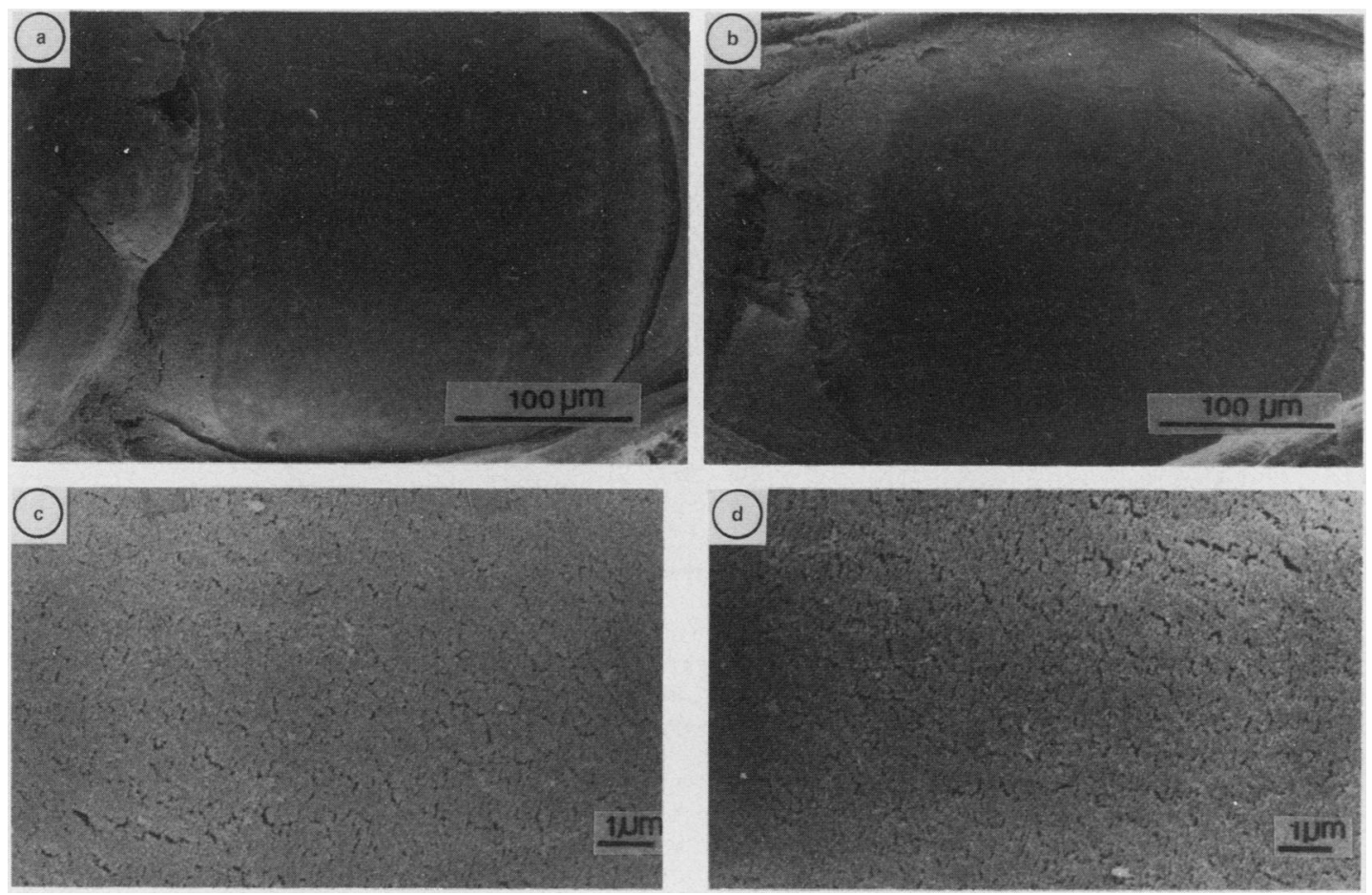

Fig. 1 (a) Surface of patella from a normal untreated mouse knee joint. (b) Surface of patella from a normal mouse knee joint immobilised for seven days. (c) Cartilage surface of untreated patella. (d) Cartilage surface of immobilised patella. Note the irregular fissuring of the surface structure. 
tised with chloral hydrate, killed by cervical dislocation, and, additionally, the patella was dissected from the arthritic joint for scanning electron microscopy. For comparison, patellae were taken from arthritic and control joints immobilised for seven days.

\section{SCANNING ELECTRON MICROSCOPY}

Patellae dissected from immobilised and nonimmobilised arthritic joints were fixed in $2 \%$ glutaraldehyde in $0 \cdot 1 \mathrm{M}$ phosphate buffer $\mathrm{pH} 7.4$ for two hours at room temperature. Subsequently the patellae were washed. After washing, the patellae with surrounding tissue were fixed in $1 \%$ osmium tetroxide in $0.1 \mathrm{M}$ phosphate buffer for one hour and subsequently washed. In addition, the patellae were dehydrated in ethanol $(30,50,70,96$, and $100 \%)$, dried to the critical point (Balzers Union, M9202), and sputtered with gold using a Balzers Union sputter apparatus (type Tpg031). The patellae were examined with a scanning electron microscope (JSM-U3 or JSM-T300).

\section{Results}

EFFECT OF IMMOBILISATION ON NORMAL JOINTS

Patellae derived from normal mouse knee joints immobilised for seven days showed some fissuring of the cartilage surface, which was more pronounced? than in the patellae of non-immobilised knee joints. (Fig. 1). Protrusions of the synovial membranes extending to the cartilage surface were not observed.

ARTHRITIC CARTILAGE

After intra-articular injection of cationic bovine serum albumin a severe arthritis developed, macros scopically characterised by erythema and oedema $\vec{P}$ Over a period of time $(1,3,7$, and 14 days afte $\vec{L}$ arthritis induction) we examined the subsequenth changes of the cartilage surface and synovial maro gins. Scanning electron micrographs showed hyper? trophy in the acute phase and after seven dayș necrosis of the synovial lining cells. The synovial $\overrightarrow{0}$ cartilage junction was the preferential site of severeo arthritic activity and gross changes in cartilage appearance (Fig. 2a). At this site many inflamma tory cells, mainly PMNs (histological observations ${ }^{15}$ ) were present in the thickened synovial membrane one and three days after arthritis induction. After 18 days less cells were observed in this area. Detritus probably disrupted cell remnants, was seen as part of an irregular meshwork of fibrinous mategi spreading out over the patellar surface. This mesh-
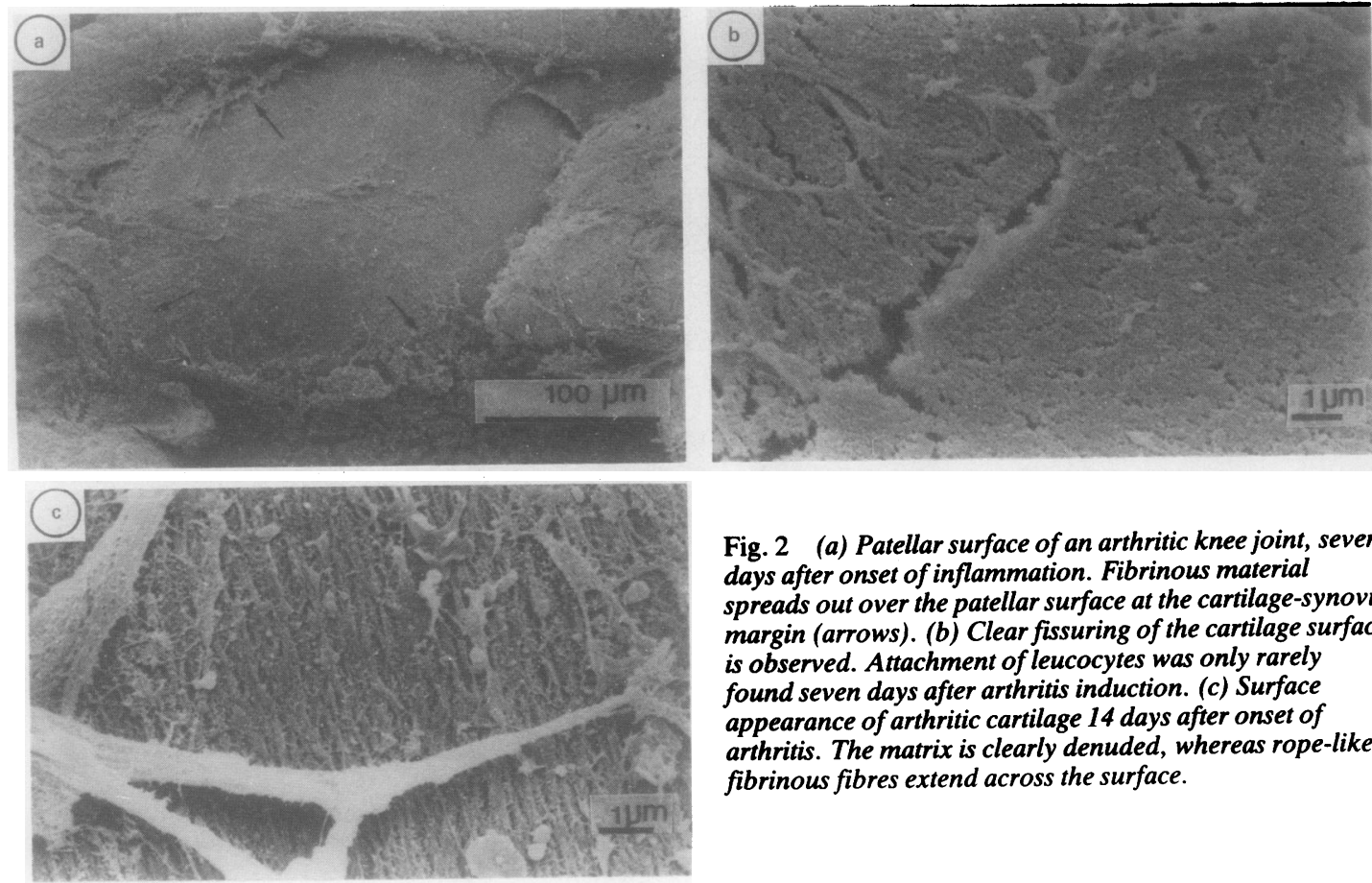

Fig. 2 (a) Patellar surface of an arthritic knee joint, seven $N$ days after onset of inflammation. Fibrinous material spreads out over the patellar surface at the cartilage-synovia's margin (arrows). (b) Clear fissuring of the cartilage surface $\mathrm{N}$ is observed. Attachment of leucocytes was only rarely found seven days after arthritis induction. (c) Surface appearance of arthritic cartilage 14 days after onset of arthritis. The matrix is clearly denuded, whereas rope-like fibrinous fibres extend across the surface. 

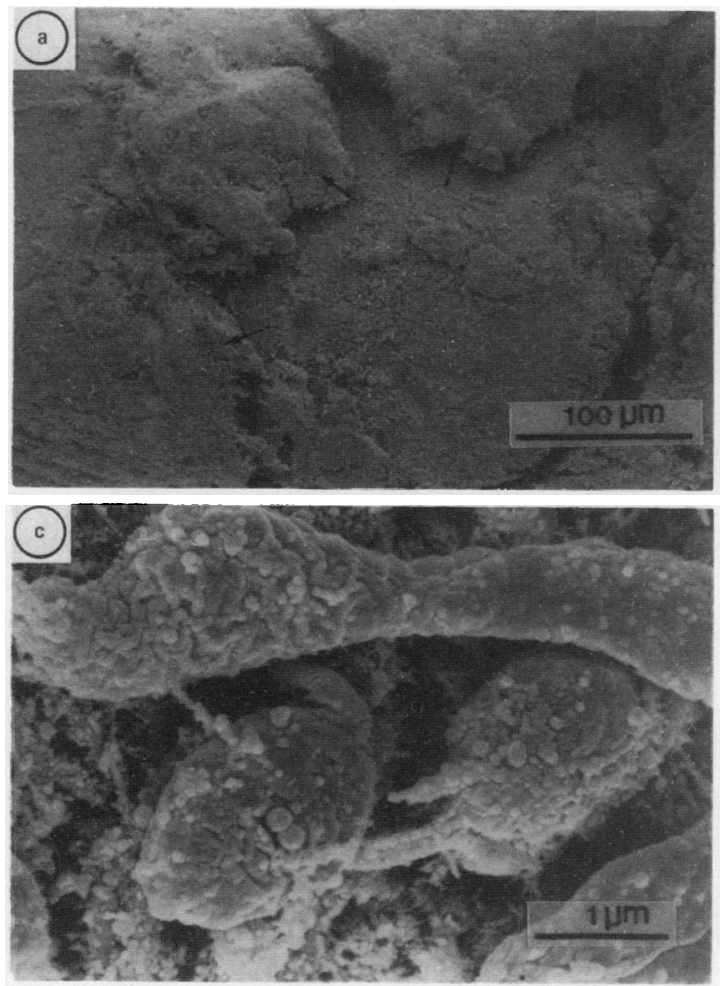

work was most extended 14 days after arthritis induction.

The patellar surface showed generalised irregularities, again mainly concentrated at the peripheral regions (Figs 2a and 2b). At higher magnifications numerous fissures of varying size and depth were seen. Size and depth of these fissures increased with time. The collagenous network of the cartilage matrix was clearly denuded after 14 days (Fig. 2c). Rope-like fibres extended across the cartilage surface in contrast with the smooth surface of normal cartilage (Fig. 1). Focusing of small amounts of intact leucocytes at the cartilage-synovium junction was only seen one day after arthritis induction. Later, attachment of cells to the cartilage surface was rarely seen.

\section{ARTHRITIC CARTILAGE FROM}

IMMOBILISED JOINTS

Short term immobilisation (seven days) of arthritic joints leads to attachment of large amounts of leucocytes to the cartilage surface as was determined by light microscopy (van Lent et al, unpublished data). Scanning micrographs showed that the patellar surface was coated with a thick mat of fibrinous

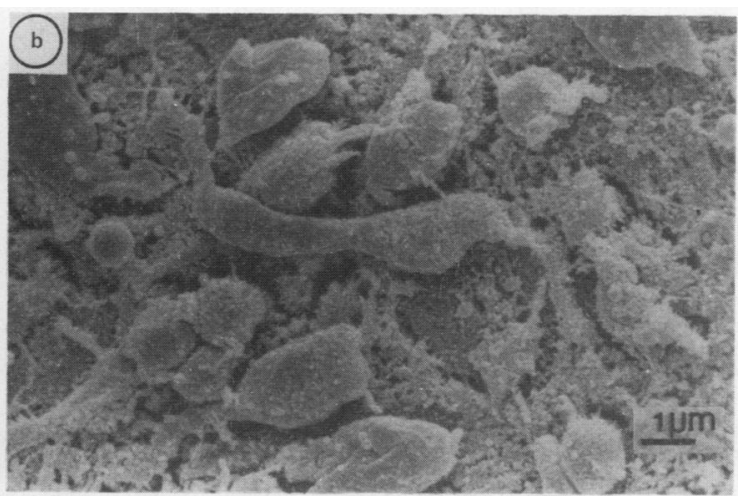

Fig. 3 (a) Patellar surface of an arthritic knee joint immobilised for seven days. Thick mats of fibrinous material cover the surface (arrows). (b) Leucocytes, surrounded by fibrinous material, attach to the cartilage surface in large amounts. (c) The attached leucocytes show marked variations in their cell surface morphology.

material extending from the synovial membrane (Fig. 3a). At higher magnification it appeared to be an extensive network of fibres in which probably cellular debris and intact leucocytes were present in large amounts. The leucocytes which covered the cartilage surface showed marked variations in cell

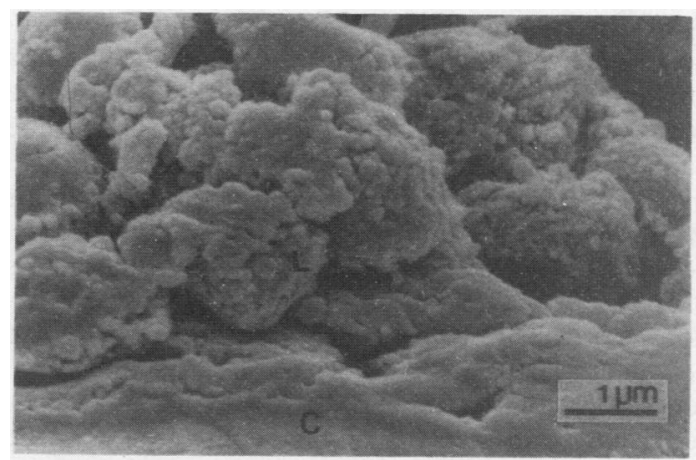

Fig. 4 Cross section of an immobilised arthritic patella. Close proximity of leucocytes to cartilage surface. No clear penetrations of membrane protrusions were observed. $C=$ cartilage $;=$ leucocytes. 
surface morphology, ranging from a rounded smooth appearance to an extensively ruffled architecture (Figs $3 b$ and c).

Scanning micrographs of cross sections of immobilised arthritic patellae showed that the cells were in close proximity to the cartilage surface (Fig. 4). Clear penetrations of membrane protrusions in the damaged cartilage matrix were hardly seen. The surface of the patellar cartilage derived from immobilised arthritic joints appeared to be more eroded than cartilage from non-immobilised arthritic joints. In particular, fissures in the cartilage surface were more pronounced.

Extensive attachment of leucocytes and increased damage were not limited to patellar cartilage but were also observed by light microscopy with tibial and femoral articular cartilage (unpublished results).

\section{Discussion}

In this study mouse patellae exposed to a chronic immune inflammatory reaction showed provoked damage of the cartilage surface with increasing time. More damage was induced in arthritic joints immobilised for a short time than in non-immobilised joints, and attachment of large amounts of inflammatory cells to the cartilage surface was evident.

Articular cartilage in rheumatoid arthritis undergoes erosion both at the synovium-cartilage junction and on the free surface of the cartilage. Erosions of the free cartilage surface were also observed in our model. These features were more pronounced in the chronic phase than in the acute phase. The collagen network was clearly denuded already one week after the start of the arthritis. Probably, depletion of proteoglycans, which is obvious in this model. ${ }^{11}$ uncovers the collagen fibres.

At the moment there is no general agreement about the part played by PMNs in cartilage destruction, some investigators suggesting that their role is only minor. Evidence was found that the destructive potential was mainly derived from fibroblast-like pannus cells which overgrow the cartilage surface from the margins. Factors like collagenase ${ }^{1}$ or interleukin $1^{2}$ could be potential candidates. Others, however, suggest that especially away from pannus PMNs may play a major part in cartilage destruction. A clear morphological relation was found between cartilage destruction and PMN presence. $^{34}$ In rheumatoid arthritis PMNs are a consistent and major cellular constituent of the inflammatory effusion, and deleterious changes in the hyaline cartilage surfaces may be due to PMN interaction. Elastase released by the PMNs may be an important denominator for cartilage destruc- tion. ${ }^{5}$ Normal PMNs have been shown to be focused to cartilage and become phagocytically active if added to cartilage derived from an antigen induced arthritic joint or rheumatoid arthritis carti-c) laginous tissues. ${ }^{89}$

Despite the potential importance of cartilage damage caused by interaction of PMNs with cartil- $\frac{\bar{s}}{\sqrt{5}}$. age surfaces, no clear cut in vivo data of these $\stackrel{\mathbb{D}}{\Omega}$ interactions are available. Biopsied specimens ofo cartilage from patients with active rheumatoide arthritis seldom show PMNs on articular surfaces. ${ }^{16}$ Similar observations are made in antigen induced $\vec{\omega}$ arthritis models. ${ }^{417}$ In our model cationic bovineo serum albumin is injected in the knee joint of immunised mice and immune complexes are clearly present on the cartilage surface. A longlasting: arthritis develops, characterised by the influx pre- $-\overrightarrow{0}$ dominantly of granulocytes. Clear focusing of cellsc్ on cartilage surfaces was never seen. When these ${ }_{0}^{N}$ arthritic joints are given a short rest, however, large numbers of cells, mainly PMNs, attach to the $\vec{P}$ cartilage surface. To our knowledge this is the firsto report which visualises attachment of numerous? leucocytes to cartilage surfaces. The same adherence pattern was found by light microscopy (dafa $\overrightarrow{0}$ not shown), indicating that the observed PNO 00 attachment was not due to artefacts introduced the method used for preparing cartilage specimeno for scanning electron microscopy.

The close proximity of inflammatory cells ando

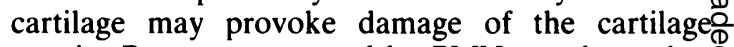
matrix. Proteases secreted by PMNs, such as elas 0 tase and cathepsin G, may escape from loca $\vec{E}$ inhibitors which are present in the synovial fluid $\beta$ in large amounts. ${ }^{16}$ In our model immobilisation clearly induced fissuring of the cartilage surface? These fissures, variable in size and depth, mayo further benefit the attachment of PMNs by creating a larger contact area, giving better hold for the 3 PMNs. In addition, immune complexes present in the superficial layers of cartilage, and thought to play an important part in mediating attachment of PMNs to cartilaginous tissues, ${ }^{12}$ may be more attainable to the activated PMNs. The numerous extended protrusions of the PMNs suggest that some. form of activation of the cell membrane has occurred. Apart from immune complexes, solublê, mediators such as $\mathrm{C} 5 \mathrm{a}^{18}$ or monokines such as interleukin $1^{19}$ may also contribute to the activated state of the PMNs. Activated PMNs show large protrusions, which may penetrate the cartilage matrix. In vitro studies indeed showed deep pene tration of PMN membrane protrusions in the cartil $-?$ age matrix as well as signs of degranulation. ${ }^{89}$ Cross sections of our immobilised arthritic patellae showed leucocytes lying close to the cartilage sur $\frac{\text { P }}{\mathbb{Q}}$ 
face, but penetration of PMN membrane protrusions was scarcely seen. This was confirmed by transmission electron microscopy (data not shown).

This study clearly indicates that the pronounced interaction between inflammatory cells and articular collagenous tissues occurs at times of rest. This interaction may lead to increased damage of the articular cartilage. A light microscopic study, in which arthritic joints were immobilised for five and seven days, showed apart from PMN attachment significantly more matrix degradation, whereas two weeks after removing the cast more chondrocyte death and osteophyte formation was observed (manuscript in preparation). Immobilisation of an arthritic joint is used in hospital to relieve pain, but these studies suggest that further assessment should be made of its effects, which may at the same time be potentially damaging to the articular cartilage.

\section{References}

1 Woolley D E, Crossley M J, Evanson J M. Collagenase at sites of cartilage erosion in the rheumatoid joint. Arthritis Rheum 1977; 20: 1231-9.

2 Pettipher E R, Henderson B, Moncada S, Higgs G A. Leucocyte infiltration and cartilage proteoglycan loss in immune arthritis in the rabbit. BrJ Pharmacol 1988; 95: 169-76.

3 Mohr W, Wild A, Wolt H P. Untersuchungen zur Pathogenese der entzundlichen Knorpelzerstorung bei der Adjuvans Arthritis der Ratte. $Z$ Rheumatol 1980; 39: 212-22.

4 Cooke T D V, Maeda M. Mechanisms of cartilage destruction in experimental complex and rheumatoid arthritis (RA). Transactions of the 26th annual meeting of the Orthopedics Research Society 1980; 5: 269.

5 Keiser H, Greenwald K, Feinstein G, Janoff A. Degradation of cartilage proteoglycan by human leucocyte granule proteases. A model of joint injury. II. Degradation of isolated bovine nasal cartilage proteoglycan. $J$ Clin Invest 1976; 57: 625-32.

6 McCormick J R, Harkin M M, Johnson K J. Suppression by superoxide dismutase of immune-complex induced pulmonary alveolitis and dermal inflammation. Am J Pathol 1981; 102: 55-61.

7 Schalkwijk J, Van den Berg W B, Van de Putte L B A, Joosten L A B. Hydrogen peroxide suppresses the proteoglycan synthesis of intact articular cartilage. J Rheumatol 1985; 12: 205-10.

8 Ugai K, Ziff M, Jasin H E. Interaction of polymorphonuclear leukocytes with immune complexes trapped in joint collagenous tissues. Arthritis Rheum 1979; 22: 353.

9 Ugai K, Ishikawa $\mathrm{H}$, Hirohata $\mathrm{K}$, Shirane $\mathrm{H}$. Interaction of polymorphonuclear leukocytes with immune complexes in rheumatoid articular cartilage. Arthritis Rheum 1983; 26: 1434-41.

10 Van den Berg W B. Van de Putte L B A, Zwarts W A, Joosten L A B. Electrical charge of the antigen determines intraarticular antigen handling and chronicity of arthritis in mice. $J$ Clin Invest 1984; 74: 1850-9.

11 Van den Berg W B, Kruijsen M W M, Van de Putte L B A, Van Beusekom H J, Van der Sluis-van der Pol M, Zwarts W A. Antigen-induced and zymosan-induced arthritis in mice: studies on in vivo cartilage proteoglycan synthesis and chondrocyte death. Br J Exp Pathol 1981; 62: 308-16.

12 Cooke T D V, Sumia M, Elliott S, Maeda M. Immune complex mediated destruction of cartilage in antigen-induced arthritis in rabbits. J Rheumatol Suppl 1983; 11: 103-7.

13 Danon D, Goldstein L, Marikovsky Y, Skutelsky E. Use of cationized ferritin as a label of negative charges on cell surfaces. J Ultrastruct Res 1972; 38: 500-10.

14 Loermans H, Wirtz P. Inhibition of the expression of pathology in dystrophic mouse leg muscles by immobilization. Br J Exp Pathol 1983; 64: 225-30.

15 Van Lent P L E M, van den Berg W B, Schalkwijk J, van de Putte L B A, van den Bersselaar L. Allergic arthritis induced by cationic antigens: relationship of chronicity with antigen retention and T cell reactivity. Immunology 1987; 62: 265-72.

16 Schalkwijk J, Van den Berg W B, Van de Putte L B A, Joosten L A B. Elastase secreted by activated polymorphonuclear leucocytes causes chondrocyte damage and matrix degradation in intact articular cartilage: escape from inactivation by alpha-1proteinase inhibitor. Br J Exp Pathol 1987; 68: 81-8.

17 Schalkwijk J, Van den Berg W B, Van de Putte L B A, Joosten L A B, Van der Sluis M. Effects of experimental joint inflammation on bone marrow and periarticular bone. A study of two types of arthritis, using variable degrees of inflammation. $\mathrm{Br} \mathrm{J}$ Exp Pathol 1985; 66: 435-44.

18 Arnaout A M, Hakim R M, Tod R F, Dana M, Colton H R. Increased expression of an adhesion-promoting surface glycoprotein in the granulocytopenia of hemodialysis. $N$ Engl J Med 1985; 312: 457-62.

19 Bates E J, Kowenko I C, Ferrante A. Conditioned medium from stimulated mononuclear leucocytes potentiates the ability of human neutrophils to damage human articular cartilage. Ann Rheum Dis 1988; 47: 1-9. 